\title{
Ueber eine subjective Erscheinung bei Betrachtung von Contouren.
}

\author{
Von \\ Dr. G. Mayerhausen in München.
}

Von verschiedenen Autoren ist bereits eine Erscheinung beschrieben worden, welche von denselben an Gittern und parallelen Liniensystemen beobachtet wurde, ich meine das wellige oder perlenschnurartige Aussehen, welches unter gewissen Umständen die einzelnen Stäbe der Gitter resp. Linien einer entsprechenden Zeichnung darbieten.

Der erste, welchem wir eine Mittheilung hierüber verdanken, ist Purkinje. *) Derselbe beobachtete bei angestrengtem Anschanen von Parallellinien auf Kupferstichen ein Flimmern in denselben, welches näher betrachtet in einem theilweisen Finandernähern und Entfernen derselben bestand, so dass die Linien wellenförmig ersehienen. „Das Wesen der Erscheinung", - sagt Purkinje, - "liegt zum Theile in der Perspective, zum Theile in den Blendungsbildern." „In dem Axenpunkte des Sehfeldes sind die Linien etwas mehr von

*) Beobachtungen und Versuche zur Physiologie der Sime. Bd. I. Prag 1823. pag. 122. 
einander entfernt, und nähern sich einander an denen ron demselben weiter abstehenden Stellen. Wie nun der Axenpunkt im Linienfelde hin und herbewegt wird, neigen sich an den entferuteren Stellen die Linien gegen einander und entfernen sich in der jedesmaligen Mitte, indess die ihnen entsprechenden Blendungsbilder ihre Gestalt behalten und sie vielfaltig decken und durchschneiden, wodureh eine Bewegung and mannichfaltige Beugung derselben entsteht, was ihnen ein wellenförmiges Ansehen giebt." - So Purkinje's Erklïrung.

Aehnliche Versuche stellte Bergmann *) theils selbst an, theils liess er dieselben von Anderen ausfübren, indem er dazu Gitterzeichnungen benutzte, deren Striche und Zwischenräume je $1 \mathrm{Mm}$. betrugen. Oefters fand es sich, dass in einer Entfernung von mehr als 5 Meter die Untersuchten sich uber die Richtung der Stabe tänschten, besonders häufig wurde die Richtnng derselben gerade senkrecht zu der wirklichen angegeben.

Bergmann sucht die Erklärung der Erscheinung in der Anordnung der Zapfen der Fovea. Er nimmt an, dass die Zapfen der Centralgrube so neben einander geordnet sind, "ahnlich wie die Zellen des Bienenstockes sich im Querschnitt zeigen." Auf ein dementsprechendes Schema legte er in verschiedenen Richtungen Gitter, und kommt auf Grund seiner Versuche, die im Original nachgelesen werden müssen, za dem Resultat, dass die Bedingungen zum Auftreten der subjectiven Erscheinung eines Gitters, welches rechtwinklig gegen das objectiv vorhandene steht, am günstigsten sind, wenn die Bilder der Stäbe und Zwischenräume etwas mehr als $1 / 8$ der Breite der Zapfen betragen.

*) C. Bergmann, Anatomisches und Physiologisches uber die Netzhaut des Auges; in Zeitschrift finr rat. Medizin (Henle und v. Pfeufer), 3. Reihe, II. Bd. 1657. pag. 83 ff. 
Hensen*) gelangt nach seinen Untersuchungen $\mathrm{zu}$ der Annahme, dass die fragliche Erscheinung eine Folge davon sei, dass nicht die Zapfenkörper es sind, sondern nur die von Pigment umbüllten äusseren Zapfenspitzen, welche durch Licht erregt werden, und die beiläufig nach M. Schultze nur $1 / 5$ des Durchmessers der Zapfenkörper besitzen. Dieser Auffassung gemäss wäre das Gesichtsfeld der Fovea kein ununterbrochenes, sondern ein lückenhaftes, aus empfindlichen Punkten und unempindlichen Kreisen bestehend, wir wären aber im Stande, die Lücken zur Linie zu ergänzen. Zur Erläuterung finden sich mehrere schematische Figuren beigefügt.

Kurz erwähnen will ich hier noch, dass Volkmann **) diese Hypothese für unannehmbar betrachtet.

Helmholtz ***) bemerkte das Welligwerden der Stäbe an einem Gitter, dessen parallele schwarze und weisse Streifen ${ }^{13} / 24=0,4167 \mathrm{Mm}$. betrugen. In einem $\mathrm{Ab}$ stande von 1,1 bis 1,2 Meter fingen für ihn die Wellenlinien an deutlich sichtbar zu werden. Der genannte Autor giebt von der Erscheinung eine Abbildung (l. c. Fig. $102 \mathrm{~A}$ ) and sucht mit Hülfe einer zweiten beigefügten Zeichnung (1. c. Fig. 102 B) ihre Erklärung aus der: Zapfenmosair des gelben Fleckes im Sinne Bergmann's zu geben.

Eine ebenfalls hierher gehörige Erscheinung beob

*) Hensen, Ueber eine Einrichtung der Fovea centralis retinae, welche bewirkt, dass feinere Distanzen als solche, dio dem Durchmesser eines Zapfens entsprechen, noch unterschieden werden können; in Virchow's Axchiv, Bd. 34, pag. 401; und: Ueber das Sehen in der Fovea centralis; in Virchow's Archiv, Bd. 39 , pag. 475 .

**) A. W. Volkmann, Weitere Untersuchungen über die Frage, ob die Zapfen der Netzhant als Raumelemente beim Sehen fungiren; in Du Bois-Reymond's und Reichert's Archiv, Jahrg. 1866. pag. 649.

***) Physiolog. Optik. p. 217.

v. Graefe's Arohiv ftir Ophthalmologie, $\mathrm{XXX}, 2$. 
achtete Riccò*) Derselbe sah nämlich Objecte mit geradlinigen Contouren, wenn er dieselben in der Distanz des deutlichen Sehens (9 Cm. für sein rechtes Ange) gegen den Himmel oder ein gut beleuchtetes Papierblatt hielt, mit einer feinen Zähnelung. Er glaubt, dass dieses Phaenomen durch die eigenthümliche Anordnng der sechseckigen Zellen des Retinaepithels erzeugt werde.

Sehliesslich hat ganz nenerdings v. Fleischl in einem in der k. k. Gesellsehaft der Aerzte zu Wien gehaltenen Fortrage: „Zur Physiologie der Retina" - welcher Vortrag mir allerdings nur im Referat der "Wiener med. Presse"**) vorliegt - dieses an Contouren sich zeigenden Wellenphänomens Erwähnung gethan.

Es heisst 1. c.: "Er (der Vortragende) weist die Erklärung dieses Phaenomens aus der ZapfenMosaik zurück, indem er zeigt, dass die Länge und Houhe der Wellen ein Vielfaches der Grösse besitzt, die sie nach jener Erklärung haben müsste. Auch ist die Kleinheit des Gesichtswinkels, unter dem die Stäbe gesehen werden, keine Bedingung für das Zustandekommen der Erscheinung, vielmehr tritt dieselbe an jedem Contour zwischen zwei verschiedenen Helligkeiten oder Farben ein, also auch an ganz dicken und nahen Gitterm, sobald nur das Bild des Contours mit einiger Geschwindigkeit parallel mit sich selbst über die Netzhaut gleitet. Wieso aber hierbei die Wellen auftreten, vermag er nicht anzugeben." Soweit das Referat.

Zufällig hatte ich mich nun bereits ebenfalls seit längerer Zeit mit ähnlichen Versuchen beschäftigt, wobei es mir jedoch niemals gelingen wollte, eine Erscheinung

* A. Ricej, Sopra un fenomeno soggetiro di visione. Amnali ai Ottalmologia. Anno VT, pag. 547. Referat im Jaliresber. 1 . Ophthalm. Jahrg. VIII, pag. 96.

**) Das hierauf Bezügliche in No. 5, Jahrg. 1884, pag. 150. 
wahrzunehmen, die ich mit voller Sicherheit hätte auf die Anordnung der Zapfen zurückführen können, immer waren die Wellen oder Zacken viel zu gross, um eine solche Erklärung zuzulassen, eine Beobachtung, die, wie oben gesagt, ja auch von v. Fleischl gemacht wurde. Aber ebenso wollten die Erklärungen der übrigen angeführten Autoren für meine Beobachtung nicht passen.

Ueberhaupt interessirte mich das citirte Referat der "Wiener med. Presse" ausserordentlich, da meine Resultate - obgleich völlig unabhängig gefunden - mit denen des genannten Forschers, auch ausser in dem bereits genannten Punkte, noch mehrfach übereinstimmten.

Zudem bin ich noch in der Lage, für meine Beobachtung eine durchaus stichhaltige Erklärung beizubringen.

Ebenso wie $\nabla$. Fleischl fand auch ich, dass es durchaus nicht absolut nothwendig ist, dass die zum Versuche benutzten Objecte unter sehr kleinem Gesichtswinkel (d. h. aus grösserer Entfernung) gesehen werden, wenngleich unter Umständen eine angemessene grössere Entfernung die Erscheinung unbedingt deutlicher hervortreten lässt. Den Grund hiervon werden wir später kennen lernen.

Ferner hatte auch ich gefunden, dass es nicht nothwendigerweise eines Stabes oder eines aus solchen bestehenden Gitters, ja nicht einmal einer Anzahl paralleler Linien bedarf, sondern dass jeder beliebige Contour zur Beobachtung geeignet ist. Jede scharfe Grenze zwischen zwei verschiedenen Helligkeiten oder Farben, jede Kante zweier aneinander stossenden verschieden beleuchteten Flüchen, jeder Rand eines Gegenstandes, der gegen eine dahinter liegende Wand u. s. w. sich genügend abhebt, zeigt die Erscheinung. Auch ist eine centrale Fixation nicht unbedingtes Erforderniss, sondern bei einiger Uebung sieht man dasselbe auch sehr schön auf den peripheren Partien der Netzhaut. 
Selbstrerständlich zeigen, wenn verhältnissmässig schmale Objecte benutzt werden, wie Stäbe, Striche a. s. w., an denen zwei Contouren sichtbar sind, diese Objecte die Erscheinung auch an beiden Contouren, und so entstehen denn auch Figuren, wie sie \%. B. Helmholtz abgebildet hat.

Die Erscheinung tritt, besonders bei sehr heller Beleuchtung nicht immer gleich auf den exsten Blick ein, sondern bisweilen erst nach kürzerem oder längerem Hinschauen.

Ein für die Erklärung sehr wichtiger Umstand jedoch, den ich bei keiner der von den genannten Autoren beschriebenen Erscheinungen genügend betont finde, wenn auch zu ersehen ist, dass derselbe dann und wann wohl beobachtet wurde, ist folgender.

Betrachten wir entweder an dem Stabgitter oder einer passenden Zeichnung oder einer beliebigen Kante zweier aneinander stossenden verschieden beleuchteten Flächen aufmerksam die Erscheinung, so wird es uns nieht entgehen, dass die Wellenlinien durchaus nicht stabile sind, und keineswegs unverändert bleiben, sondern dass ein fortwährender Wechsel in dem Phaenomen sich derart vollzieht, dass wir an derselben Stelle, wo kurz vorher ein Wellenberg sich befand, im nächsten Moment ein Wellenthal sehen und umgekehrt, auch wenn wir die Augen absolut ruhig halten. Die Wellen sind auch durchaus nicht so regelmässig, sondern bald kürzer, bald länger gestreckt, bald wieder mehr spitz, einer Zähnelung zu vergleichen, bald abgerundet; kurz wir sehen, auch die Wellen als solche wechseln fortwährend ihre Grösse und Form.

Dieselbe Erscheinung mit ihrem fortwährend wechselnden Aussehen zeigt sich aber auch im Nachbilde, und zwar sowohl im entoptischen als im ectoptischen, gleichviel ob eine centrale oder excentrische vorherige Betrachtung des Objectes stattfand. 
Eine Erklärung dieser Erscheinung ist bei einer aufmerksamen Beobachtung nicht schwer $\mathrm{zu}$ finden in den eigenthümlichen fortwährend wechselnden Schwankangen des Eigenlichtes der Netzhaut.

Bekanntlich ist ja unser Gesichtsfeld auch bei vollständigem Abschlusse alles objectiven Lichtes und ohne jeden anderen Reiz von aussen nicht absolut dunkel. Es bleibt sich dabei vollständig gleich, ob wir in einem hellen Raume die Augen genügend bedecken - ohne natürlich im geringsten einen Druck auf dieselben auszuüben oder $o b$ wir uns mit geöffneten Augen in einem absolut finsteren Zimmer befinden.

Haben wir uns auf diese Weise von dem Vorhandensein des Eigenlichtes der Netzhaut einmal uberzeugt, so wird es uns übrigens nicht schwer fallen, dasselbe auch bei geöffneten Augen ohne vollständigen Abschluss des äusseren Lichtes wahrzunehmen.

Sehen wir z. B. von einem erleuchteten Orte aus durch die geöffnete Thür in ein finsteres Zimmer hinein, so bemerken wir, dass der von der Thüröffnung begrenzte dunkle Raum durchaus nicht absolut lichtlos ist. Wir sehen daselbst, und zwar im ganzen Bereiche des Gesichtsfeldes, ein unausgesetztes "Kriebeln und Wiebeln", nicht einen Moment herrscht an derselben Stelle eine vollkommene Ruhe. Je länger wir hinschauen und je mehr wir unsere Aufmerksamkeit der Sache zuwenden, desto deutlicher und stärker tritt das Eigenlicht hervor. Bald möchte man es einen äusserst sanft phosphorescirenden Staub nennen, der nach den verschiedensten Richtungen in nicht genau $\mathrm{zu}$ verfolgenden Bahnen durcheinander strömt, bald würde man wiederum das Aussehen als ein ausserordentlich zart und fein marmorirtes bezeichnen, dessen Muster jedoch durch Auftauchen und Wiederverschwinden von lichten und lichtlosen Theilchen in jedem Augenblicke sich anders gestaltet. 
Dazwischen erscheinen bei manchen Mensehen oft hier und da Bruchstücke der von mir*) als subjective Wahrnehmung des Faserverlaufs in der Nervenfaserschicht der Retina beschriebenen Figur.

Ganz ebenso zeigen sich diese Erscheinungen des Eigenlichtes beim Blick auf irgend eine beliebige dunkle Fläche. Je heller diese letztere gewählt wird, desto weniger Bestimmtes lässt sich unterscheiden, so dass bei einer gewissen Helligkeit sich nur noch eine eigenthümliche Unruhe im Gesichtsfelde geltend macht, ohne dass man jedoch von dem fortwährenden Wechsel zwischen helleren und dunkleren Stellen und Punkten sich noch deutlich Rechenschaft geben könnte.

Es wird uns übrigens dies nicht verwundern, wenn wir bedenken, dass das Eigenlicht der Netzhaut ja nur von sehr schwacher Intensität ist. Beiläufig sei bemerkt, dass die Stärke desselben von Volkmann **) nach seinen Versuchen angegeben wird als gleich der Helligkeit einer schwarzen Sammetfläche, beleuchtet durch eine Stearinkerze in 9 Fuss Entfernung; A ubert***) bestimmte dieselbe im lichtlosen Raume als gleich der Helligkeit, welche ein weisses Papier hat, wenn es von der Hälfte der Lichtstärke der Venus zur Zeit ihres höehsten Glanzes beleuchtet wird.

Un sich nun von der Richtigkeit meiner Exklarung der in Rede stehenden Erscheinung zu abbrzengen, wählt man am besten eine nicht sehr helle Beleuchtung also entweder eine künstlich herabgesetzte oder einfach die Dämmerung, - da, wie bekannt, bei geringerer Helle das Eigenlicht besser sichtbar ist. Wir brauchen uns

*) Ueber eine eigenthümliche Erscheinungsform des Eigenliehtes der Netzhaut, nebst Bemerlung über die Gleichgewichtslage der Bulbi im wachen Zustande; im Archiv $f_{*}$ Augenheilk. Bd. XIII, pag. 77.

**) efr. HeImholtz, Physiolog. Optik. pag. 313.

***) In Graefe-Saemisch, Handb. Bd, II, pag. 486. 
auch gar keiner speciell ad hoc hergerichteten Apparate zu bedienen, sondern können jeden beliebigen Gegenstand mit scharfem Contour benützen.

Betrachten wir also bei genügend verminderter Beleuchtung z. B. an irgend einem im Zimmer befindlichen Möbelstücke eine Kante, in welcher verschieden helle Flächen zusammenstossen, aus einer Entfernung von beiläufig 2 bis 3 Meter, so werden wir zunächst sehr schon bemerken, wie diese Kante fortwährend in der besprochenen Weise ihr Aussehen ändert, und zwar ist das Entstehen aller dieser Verwandlungen auf das deutlichste $\mathrm{zu}$ verfolgen. Ausserdem aber, wenn wir nun recht genau zusehen, gewahren wir zu gleicher Zeit im ganzen übrigen Gesichtsfelde sowohl, als auch speciell in der ganzen Ausdehnung der Kante, welche die Grenze zwischen der dunkleren und helleren Fläche bildet, die beschriebenen fortwährenden örtlichen Intensitätsschwankungen des Eigenlichtes, und es gehört keine grosse Uebung in der Beobachtung daza, um sich zur Genüge davon zu überzengen, dass diese Kante in allen ihren Theilen auch genau entsprechend diesen Schwankungen des Eigenlichtes continuirlich in der bekannten Weise eine Veränderung ihres Aussehens erfährt.

Ich möchte hier nochmals darauf aufmerksam machen, dass je länger man die Betrachtung fortsetzt und je mehr man seine Aufmerksamkeit auf dieselbe richtet, die Erscheinung in ihrem ganzen $\mathrm{Zu-}$ sammenhange desto klarer und deutlicher sich präsentirt. *)

*) Wird der Versuch in einem nur von dem ganz matten Scheine einer Lampe möglichst wenig erhellten Zimmer vorgenommen, und als Versuchsobject ein 1 bis $2 \mathrm{Cm}$. breiter weisser Streifen auf dunklem Grunde (oder umgekehrt) in beiläufig 2 oder 3 Meter betrachtet, so sieht man zuerst sehr schön, wie nur die Ränder des Streifens (also die Grenzen zwischen Hell und Dunkel) diese Veränderung zeigen, bei längerem Hinblicken aber wird das 
Genau dasselbe sieht man natïrlich anch an jedem anderen Contour, an jedem scharfen Rande, jedem Stabe, event. einem aus solchen bestehenden Gitter, an jedem Striche resp. einer aus solchen zusammengesetzten Zeichnung.

Dass wir in einer gewissen Entfernung die Erscheinung besser wahrnehmen, als in unmittelbarer Nähe, hat darin seinen Grund, dass uns eben jede subjective Gesichtstrscheinung bei Fixation auf eine weitere Distanz in allen ihren Einzelheiten grösser und daher auch deutlicher erscheints

Wenn wir den Versuch mit parallelen Liniengruppen anstellen, so werden wir natürlich für den Fall, dass dieselben sehr fein gewählt werden, dieselben näher halten müssen als stärkere, da unsere Sehschärfe uns nicht gestattet, in grösserer Entfernung die Linien als einzelne zu differenziren, wir also dann anch ihre einzelnen Contouren nicht mehr unterscheiden. Da wir dann die Beobachtung aus viel grösserer Nähe machen müssen, präsentiren sich aus dem eben angeführten Grunde die Détails der Erscheinung natürlich auch in viel kleinerem Maassstabe, und vor allen Dingen ist es der fortwährende Wechsel im Aussehen der Contouren, welcher dann selbst bei grösster Aufmerksamkeit, wenn überhaupt noch mit Sicherheit, so doch nur ausserordentlich schwierig zu arkennen ist. -

Nach allen diesen Auseinandersetzungen drängt sich unwillkürlich die Frage auf: Sind nicht vielleicht alle diese Erscheinungen, wenigstens im Grunde genommen, identisch? und sollte sich nicht für dieselben, wenn auch vielleicht mit Modificationen, dieselbe Erklärung geben lassen?

München, Februar 1884.

Eigenlicht im ganzen Gesichtsfelde so ausserordentlich stark sichtbar, dass es das Bild des Streifens vollkommen ,überwuchert", wnd dieser daher ganz verschwindet. 